\title{
Síndrome respiratória aguda grave em indígenas no contexto da pandemia da COVID-19 no Brasil: uma análise sob a perspectiva da vigilância epidemiológica
}

\author{
Severe acute respiratory syndrome in indigenous people in the \\ context of the COVID-19's pandemic in Brazil: an analysis from the \\ perspective of epidemiological surveillance
}

\author{
William Nicoleti Turazza \\ da Silva
}

Maria Fernanda Prado Rosa

Kaio Saramago Mendonça (iD)

Giulia de Assis Queiroz

Stefan Vilges de Oliveira* (iD)

Faculdade de Medicina, Universidade Federal de Uberlândia, Uberlândia, MG, Brasil

\section{* E-mail: stefanbio@yahoo.com.br}

Recebido: 30 ago 2020

Aprovado: 24 set 2020

\begin{abstract}
RESUMO
Introdução: As populações indígenas são mais vulneráveis a infecções respiratórias e enfrentam situações que podem agravar a evolução e o prognóstico da COVID-19. Nesse contexto, identificar os grupos expostos a maior risco e propor estratégias de predição, prevenção e controle são as premissas da vigilância epidemiológica. Objetivo: Analisar o impacto da pandemia da COVID-19 na população indígena brasileira a partir das internações por síndrome respiratória aguda grave (SRAG). Método: Estudo epidemiológico, descritivo e quantitativo dos casos de SRAG em pacientes autodeclarados indígenas notificados ao Sistema de Informação de Vigilância Epidemiológica da Gripe (SIVEP-Gripe) da Secretaria de Vigilância em Saúde do Ministério da Saúde (SVS/MS) do Brasil, de $1^{\circ}$ de janeiro a 16 de junho de 2017, 2018, 2019 e 2020. Resultados: 0 total de casos de SRAG foi de 688, com 318 confirmados para a COVID-19. Dos pacientes com a SRAG, 237 evoluíram com alta e 211 com óbito. Para a COVID-19, 81 evoluíram com alta e 155 com óbito. Casos e óbitos por SRAG e COVID-19 predominaram no sexo masculino. Houve pico de casos e óbitos entre os menores de 1 ano e entre maiores de 50 anos. Para SRAG, predominaram casos e óbitos rurais e para COVID-19, urbanos. Houve predomínio de casos da SRAG e COVID-19 nos estados do Amazonas, São Paulo e Pará. Já os óbitos predominaram nos estados do Amazonas, Pará e Roraima. Conclusões: As populações ficam sujeitas a situações de maior vulnerabilidade durante a pandemia, constituindo risco para suas saúdes e para o seu patrimônio. Mais pesquisas e ações de vigilância epidemiológica efetivas voltadas para essa população se mostram essenciais.
\end{abstract}

PALAVRAS-CHAVE: COVID-19; Epidemiologia; Pandemia; Saúde Indígena, Vigilância em Saúde

\section{ABSTRACT}

Introduction: Indigenous populations are more vulnerable to respiratory infections and face situations that can worsen the evolution and prognosis of COVID-19. In this context, identifying the groups exposed to the greatest risk and proposing strategies for prediction, prevention and control are the premises of Epidemiological Surveillance. Objective: To analyze the impact of COVID-19's pandemic on the Brazilian indigenous population considering hospitalizations for Severe Acute Respiratory Syndrome (SARS). Method: Epidemiological, descriptive and quantitative study of SARS cases in self-declared indigenous patients notified to the Influenza Epidemiological Surveillance Information System (SIVEPGripe) of the Health Surveillance Secretary of the Ministry of Health (SVS/MS) of Brazil, from 01/01 to 16/06 of 2017, 2018, 2019 and 2020. Results: The total number of SARS cases was 688 , with 318 confirmed for COVID-19. Of the patients with SARS, 237 evolved with discharge and 211 with death. For COVID-19, 81 evolved with discharge and 155 with death. Cases and deaths by SARS and COVID-19 were predominated in males. The cases and deaths were predominant among children under 1 year old and among those over 50 years old. For 
SARS, rural cases and deaths predominated and, for COVID-19, the urban ones. There was a predominance of cases of SARS and COVID-19 in the states of Amazonas, São Paulo and Pará. Deaths predominated in the states of Amazonas, Pará and Roraima. Conclusions: The populations are subjected to situations of greater vulnerability during the pandemic, representing a risk to their health and their cultural heritage. More research and effective epidemiological surveillance actions aimed at this population are essential.

KEYWORDS: COVID-19; Epidemiology; Pandemics; Health of Indigenous Peoples, Health Surveillance

\section{INTRODUÇÃO}

A pandemia causada pela COVID-19 trouxe consequências graves a todos os sistemas de saúde do mundo, incluindo o brasileiro, principalmente no que diz respeito às populações marginalizadas ${ }^{1}$. Os focos iniciais de transmissão da COVID-19 no país foram os estados de São Paulo (SP) e Rio de Janeiro (RJ), rapidamente se espalhando pelo restante das capitais, de modo que, ao final de março de 2020, a maioria delas já registrava casos da doença1. Meses depois, ao final de agosto, ainda se pode notar um importante destaque da região Sudeste quanto ao número de casos e óbitos pela COVID-19², entretanto, apesar dessa predominância regional do sudeste, a região da Amazônia Legal, que compreende uma grande parcela dos povos indígenas e 24 Distritos Sanitários Especiais Indígenas (DSEIS), concentra aproximadamente $1 / 5$ dos casos e óbitos do país ${ }^{3}$.

De acordo com o censo demográfico de $2010^{4}, 896$ mil indivíduos se declararam como indígenas, dos quais aproximadamente $64 \%$ residiam em áreas rurais. As populações indígenas de todo o mundo são extremamente mais vulneráveis a infecções respiratórias, que podem evoluir, assim como a COVID-19, para síndrome respiratória aguda grave (SRAG), mesmo fora dos períodos epidêmicos, sendo uma das principais causas de morbimortalidade entre esses indivíduos ${ }^{1}$. A Fundação Oswaldo Cruz ${ }^{1}$ destacou que "diferentes vírus, como os do sarampo, da varíola e da influenza, levaram a grandes epidemias e até ao extermínio de alguns povos nativos no Brasil”.

Apesar de alguns povos indígenas serem considerados isolados, vários deles frequentemente se conectam com áreas urbanas $\mathrm{e}$ mantêm vínculos econômicos e de serviço, principalmente no estado do Amazonas, que concentra grande parte dessa população, havendo, portanto, alto potencial de espalhamento do vírus entre esses indivíduos ${ }^{1,5}$. Ressalta-se, ainda, que o estado amazonense já chegou a atingir o marco de segundo maior em número de casos confirmados da COVID-19 e o maior em número de óbitos, ocupando atualmente a nona posição em casos confirmados e a sétima em total de óbitos dentre outras unidades federativas nacionais ${ }^{6}$.

Segundo a Associação Brasileira de Saúde Coletiva (ABRASCO), estudos confirmaram situações importantes de vulnerabilidade que podem agravar a evolução e o prognóstico da COVID-19 entre os povos indígenas, tais como: anemia, desnutrição, malária, diabetes e obesidade ${ }^{7}$. Além disso, a geografia aparece como um outro fator agravante importante, visto que, em geral, esses povos residem em locais remotos e têm dificuldade de acessar o sistema de saúde, mais próximo de pequenos centros urbanos, com pouca ou nenhuma disponibilidade de instalações hospitalares especializadas e leitos de unidade de terapia intensiva (UTI), enquanto, nos maiores centros urbanos, os leitos estão sujeitos a períodos de superlotação, como já ocorrido, por exemplo, em Manaus ${ }^{5,7}$.

Somando-se a isso, o Brasil ainda possui uma baixa taxa de testagem populacional, de maneira que números de casos e óbitos estão sujeitos a subnotificação e não mostram o real contexto em que o país está inserido ${ }^{1}$, o qual pode ser percebido por outras formas, como o aumento exacerbado do número de mortes por SRAG. A SRAG é uma das principais complicações do vírus e é monitorada no país pelo Sistema de Informação de Vigilância Epidemiológica da Gripe (SIVEP-Gripe) da Secretaria de Vigilância em Saúde do Ministério da Saúde (SVS/MS) ${ }^{1}$.

Nesse contexto, ressalta-se que, segundo o Guia de Vigilância em Saúde de 2019, do MS, durante epidemias e surtos, deve-se buscar identificar grupos expostos a maior risco e fatores de risco, com estabelecimento de relações causais, a fim de determinar as principais características epidemiológicas da doença e condições que afetem sua propagação e as medidas de saúde empregadas no combate, de modo que, portanto, torna-se importante a realização de práticas de Vigilância em Saúde efetivas voltadas para a população indígena, desde a produção de informações técnicas até a adoção de condutas para prevenção e para controle da COVID-198,9.

Dessa forma, frente ao risco de subnotificação de casos e óbitos da COVID-19 e aos diferentes contextos de vulnerabilidade aos quais os povos indígenas estão submetidos, somando-se a situações de atribulações interétnicas, como preconceito, que dificultam ainda mais o enfrentamento à pandemia ${ }^{1}$, torna-se evidente a necessidade de melhor investigação do impacto da pandemia nessa população. Deste modo, o presente estudo teve por objetivo analisar o impacto da pandemia de COVID-19 na população indígena brasileira por meio da análise das internações pela SRAG entre esses indivíduos.

\section{MÉTODO}

Realizou-se um estudo de caráter epidemiológico, descritivo e quantitativo dos casos de SRAG em pacientes autodeclarados indígenas notificados ao SIVEP-Gripe da SVS/MS do Brasil. Foram analisados os períodos de $1^{\circ}$ de janeiro a 16 de junho dos anos de 2017, 2018, 2019 e 2020. Os dados epidemiológicos foram acessados pela Plataforma Integrada de Vigilância em Saúde (IVIS) do $M S^{10}$, que os disponibiliza de forma aberta em formato de planilha.

Em 2020, o país analisado é composto por 211.755.692 habitantes com uma densidade demográfica de $24,88 \mathrm{hab} / \mathrm{km}^{2}$ de 
acordo com estimativas populacionais do Instituto Brasileiro de Geografia e Estatística (IBGE) ${ }^{11}$. Segundo o levantamento censitário de 2010, 817.963 indivíduos se autodeclaravam indígenas ${ }^{12}$.

Neste estudo, foram considerados os casos que atenderam os critérios para preenchimento da ficha de notificação dos casos de SRAG: indivíduo hospitalizado com febre, mesmo que referida, acompanhada de tosse ou dor de garganta e que apresente dispneia ou saturação de $\mathrm{O}_{2}<95 \%$ ou desconforto respiratório ou que evoluiu para óbito por SRAG independentemente de internação ${ }^{13}$.

As variáveis analisadas foram: semana epidemiológica; unidade federativa; sexo [masculino; feminino; ignorado]; faixa etária; raça/cor do paciente [branca; parda; preta; indígena; amarela; ignorada]; zona da residência [urbana, rural, periurbana; ignorado]; internação em UTI [sim; não; ignorado]; uso de suporte ventilatório [sim; não; ignorado]; classificação final do caso [SRAG de outras etiologias; SRAG não especificada; COVID-19]; evolução do caso [cura; óbito; ignorado].

A análise de todas as variáveis foi realizada por estatística descritiva, apresentada em números brutos, e medidas de frequência. A análise de casos de 2017 a 2019 por semana epidemiológica (SE) foi feita através de média aritmética simples e medidas de mínimo (o menor número entre os três anos para a SE) e máximo (o maior número entre os três anos para a $\mathrm{SE}$ ). A incidência dos casos da SRAG foi calculada utilizando o número de casos divididos pela população indígena do levantamento censitário de 2010 e multiplicados por 100 mil habitantes, e a letalidade dos casos foi calculada utilizando o número de óbitos dividido pelo número de casos e multiplicado por 100. O software utilizado para análise de dados foi Microsoft Office 2016 Excel.

Não foram acessados dados nominais dos pacientes ou quaisquer outros que possibilitem sua identificação, visto que pesquisa foi realizada a partir de um banco de dados secundários. Dessa forma, não foi necessária a submissão ao Comitê de Ética em Pesquisa (CEP), de acordo com a Resolução do Conselho Nacional de Saúde $n^{\circ} 510$, de 7 de abril de 2016, por cumprimento das normas éticas nacionais.

\section{RESULTADOS}

O total de casos notificados da SRAG para a população indígena foi de 688, com 318 (46,22\%) confirmados para a COVID-19, 354 $(51,45 \%)$ de não identificados e outros 16 diagnosticados por outra etiologia. Dos pacientes com a SRAG, 237 (34,45\%) evoluíram com alta, 211 (30,67\%) com óbito e 240 (34,88\%) não tiveram evolução identificada. A incidência para a SRAG foi de 84,11 , enquanto a letalidade foi de 30,66. Para a COVID-19, 81 (25,47\%) evoluíram com alta, 155 (48,74\%) com óbito e 82 (25,79\%) sem evolução identificada. A incidência foi de 38,87 , enquanto a letalidade foi de 48,74 . Do total de óbitos, 73,45\% foram pela COVID-19.

O número de casos e de óbitos por SRAG foi maior para o sexo masculino. Em relação à faixa etária, houve um pico de casos $\mathrm{e}$ óbitos entre os menores de 1 ano e outro entre os maiores de 50 anos. Quanto à localização, predominaram os casos de óbitos rurais. Para a COVID-19, o sexo masculino também predominou tanto em casos, como em óbitos. Quanto à faixa etária, também houve dois picos de casos e óbitos, o primeiro entre os menores de 1 ano e o segundo para os maiores de 60 anos. Para localização, predominaram os casos e óbitos urbanos (Tabela 1).

Tabela 1. Frequência bruta e relativa dos casos e óbitos por SRAG e SRAG com COVID-19 entre indígenas, segundo sexo, faixa etária e zona de residência, de $1^{\circ}$ de janeiro de 2020 a 16 de junho de 2020, Brasil.

\begin{tabular}{|c|c|c|c|c|}
\hline & \multicolumn{2}{|c|}{ Casos } & \multicolumn{2}{|c|}{ Óbitos } \\
\hline & $\mathrm{N}$ & $\%$ & $\mathrm{~N}$ & $\%$ \\
\hline \multicolumn{5}{|l|}{ SRAG } \\
\hline \multicolumn{5}{|l|}{ Sexo } \\
\hline Masculino & 399 & $57,99 \%$ & 138 & $65,40 \%$ \\
\hline Feminino & 289 & $42,01 \%$ & 73 & $34,60 \%$ \\
\hline \multicolumn{5}{|l|}{ Faixa etária } \\
\hline$<1$ & 75 & $10,90 \%$ & 16 & $7,58 \%$ \\
\hline $1-4$ & 64 & $9,30 \%$ & 3 & $1,42 \%$ \\
\hline $5-9$ & 15 & $2,18 \%$ & 2 & $0,95 \%$ \\
\hline $10-14$ & 6 & $0,87 \%$ & 0 & $0,00 \%$ \\
\hline $15-19$ & 26 & $3,78 \%$ & 6 & $2,84 \%$ \\
\hline $20-29$ & 51 & $7,41 \%$ & 5 & $2,37 \%$ \\
\hline $30-39$ & 54 & $7,85 \%$ & 12 & $5,69 \%$ \\
\hline $40-49$ & 67 & $9,74 \%$ & 12 & $5,69 \%$ \\
\hline $50-59$ & 85 & $12,35 \%$ & 27 & $12,80 \%$ \\
\hline $60-69$ & 72 & $10,47 \%$ & 32 & $15,17 \%$ \\
\hline $70-79$ & 85 & $12,35 \%$ & 44 & $20,85 \%$ \\
\hline$\geq 80$ & 88 & $12,79 \%$ & 52 & $24,64 \%$ \\
\hline \multicolumn{5}{|c|}{ Zona de residência } \\
\hline Urbana & 309 & $44,91 \%$ & 92 & $43,60 \%$ \\
\hline Rural & 325 & $47,24 \%$ & 95 & $45,02 \%$ \\
\hline Periurbana & 8 & $1,16 \%$ & 2 & $0,95 \%$ \\
\hline Ignorados & 46 & $6,69 \%$ & 22 & $10,43 \%$ \\
\hline \multicolumn{5}{|c|}{ SRAG com COVID-19 } \\
\hline \multicolumn{5}{|l|}{ Sexo } \\
\hline Masculino & 195 & $61,32 \%$ & 107 & $69,03 \%$ \\
\hline Feminino & 123 & $38,68 \%$ & 48 & $30,97 \%$ \\
\hline \multicolumn{5}{|l|}{ Faixa etária } \\
\hline$<1$ & 16 & $5,03 \%$ & 9 & $5,81 \%$ \\
\hline $1-4$ & 6 & $1,89 \%$ & 2 & $1,29 \%$ \\
\hline $5-9$ & 3 & $0,94 \%$ & 0 & $0,00 \%$ \\
\hline $10-14$ & 1 & $0,31 \%$ & 0 & $0,00 \%$ \\
\hline $15-19$ & 6 & $1,89 \%$ & 3 & $1,94 \%$ \\
\hline $20-29$ & 16 & $5,03 \%$ & 3 & $1,94 \%$ \\
\hline $30-39$ & 21 & $6,60 \%$ & 6 & $3,87 \%$ \\
\hline $40-49$ & 42 & $13,21 \%$ & 12 & $7,74 \%$ \\
\hline $50-59$ & 51 & $16,04 \%$ & 19 & $12,26 \%$ \\
\hline $60-69$ & 45 & $14,15 \%$ & 27 & $17,42 \%$ \\
\hline $70-79$ & 60 & $18,87 \%$ & 38 & $24,52 \%$ \\
\hline$\geq 80$ & 51 & $16,04 \%$ & 36 & $23,23 \%$ \\
\hline \multicolumn{5}{|c|}{ Zona de residência } \\
\hline Urbana & 166 & $52,20 \%$ & 73 & $47,10 \%$ \\
\hline Rural & 122 & $38,36 \%$ & 65 & $41,94 \%$ \\
\hline Periurbana & 3 & $0,94 \%$ & 2 & $1,29 \%$ \\
\hline Ignorados & 27 & $8,49 \%$ & 15 & $9,68 \%$ \\
\hline
\end{tabular}

Fonte: Dados do SIVEP-Gripe da SVS/MS do Brasil, 2020.

SRAG: Síndrome respiratória aguda grave. 
A letalidade para ambas as condições teve um pico no primeiro ano de vida e mostrou correlação com o aumento da idade (Figura 1).

Para as unidades federativas brasileiras, houve predomínio de casos de SRAG e COVID-19 entre indígenas nos estados do Amazonas, São Paulo e Pará (Figura 2). Os óbitos predominaram nos estados do Amazonas, Pará e Roraima.

Em relação a 2019, em 2020 para SRAG, houve um aumento de $160,60 \%$ nos casos e de $744,00 \%$ das mortes. Enquanto a letalidade em 2019 foi de 9,4 , no ano seguinte foi de 30,66, que corresponde a um aumento de $226,17 \%$. Em relação aos pacientes que necessitaram de internação em UTI, houve um aumento de 162,74\% em relação ao ano anterior e, para suporte ventilatório, 133,33\% (Figura 3).

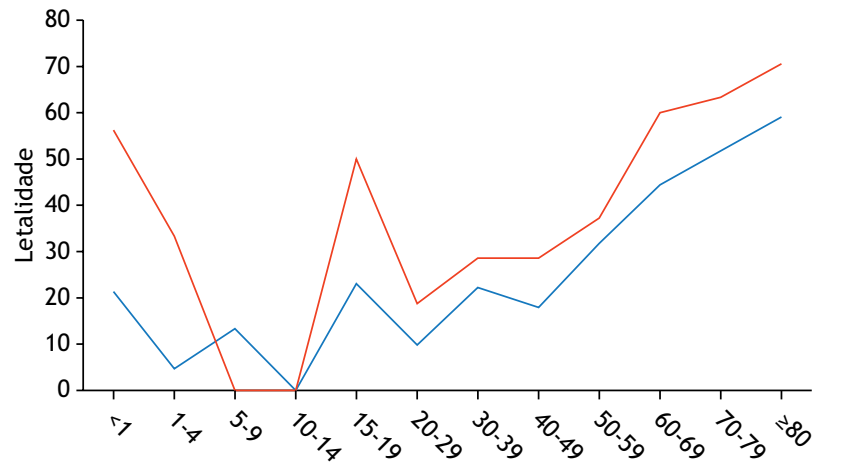

- SRAG - COVID-19

Faixa etária

Fonte: Dados do SIVEP-Gripe da SVS/MS do Brasil, 2020.

Figura 1. Letalidade segundo a faixa etária dos casos de síndrome respiratória aguda grave (SRAG) e SRAG com COVID-19, entre indígenas, de 1 de janeiro de 2020 a 16 de junho de 2020, Brasil.

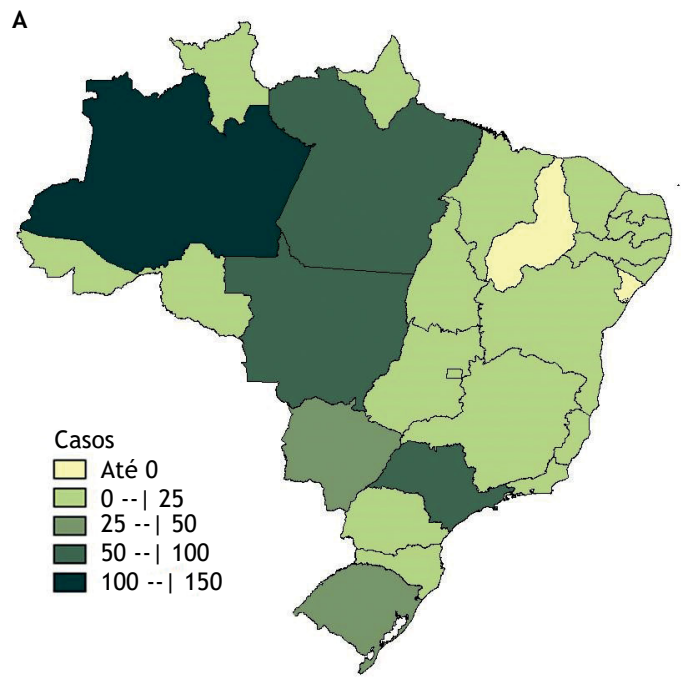

Do total de pacientes que necessitaram de internação em UTI em 2020, 43,28\% foram confirmados para COVID-19. Para a necessidade de suporte ventilatório, a porcentagem para essa doença correspondeu a $46,64 \%$.

A partir da SE 13, os casos de SRAG foram expressivamente maiores que nos anos anteriores, acompanhados do crescimento dos casos da COVID-19. Em comparação aos anos anteriores, os casos de SRAG não confirmados para COVID-19 também superam a média (Figura 4).

\section{DISCUSSÃO}

Analisando o total de casos da população brasileira, houve 291.130 casos de SRAG em 2020, com 67.618 óbitos, sendo desses casos 117.432 positivos para a COVID-19, com 14.275 óbitos, o que representa aumento expressivo no número de casos e óbitos pela SRAG, acompanhados de casos e óbitos da COVID-19, quando comparados aos anos anteriores. É interessante notar (Figura 4) que os casos de SRAG, incluindo aqueles não confirmados para a COVID-19, também tiveram um evidente aumento entre indígenas.

Como explicitado por França et al. ${ }^{14}$, a causa básica da morte (CB), definida como a circunstância do acidente ou doença que iniciou a cadeia de eventos que levou à morte, pode ser diversa no contexto atual da pandemia da COVID-19, passando por SRAG, pneumonia, como caracterizado inicialmente nos casos na China, e insuficiência respiratória, sendo observado aumento recente dessas patologias datadas como causa de óbito em várias capitais brasileiras ${ }^{14,15,16}$.

No contexto da atual pandemia da COVID-19, é possível observar, principalmente em capitais brasileiras, o aumento dos registros de patologias como SRAG, pneumonia e insuficiência

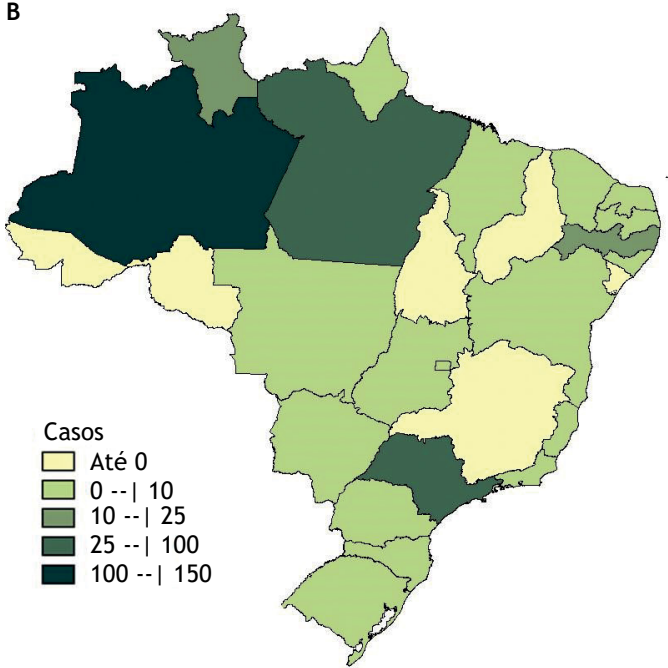

Fonte: Elaborada pelos autores com base nos dados do SIVEP-Gripe da SVS/MS do Brasil, 2020.

Figura 2. A: Número de casos notificados de síndrome respiratória aguda grave (SRAG) por unidade federativa ao Sistema de Informação de Vigilância Epidemiológica da Gripe (SIVEP-Gripe) da Secretaria de Vigilância em Saúde do Ministério da Saúde (SVS/MS) do Brasil entre indígenas. B: Número de casos notificados de SRAG com a COVID-19 por unidade federativa ao SIVEP-Gripe da SVS/MS do Brasil entre indígenas. Período de $1^{\circ}$ de janeiro de 2020 a 16 de junho de 2020 . 


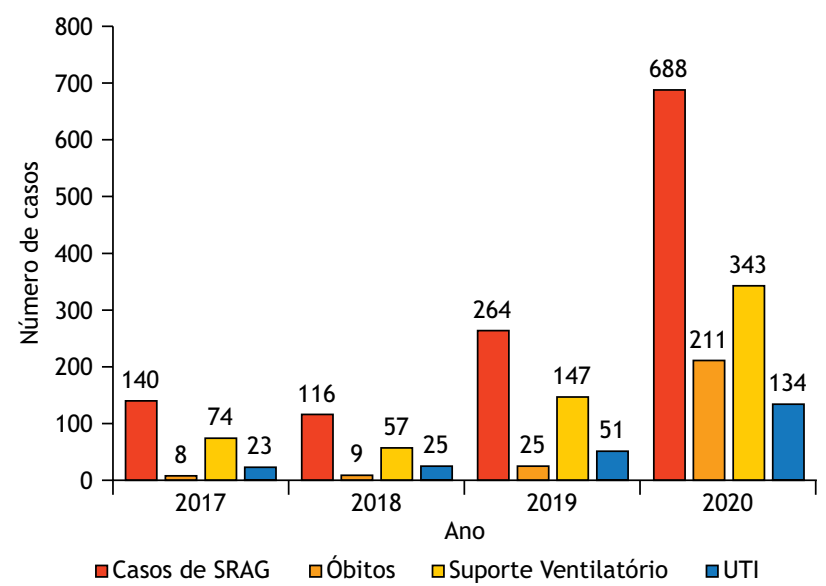

Fonte: Dados do SIVEP-Gripe da SVS/MS do Brasil, 2020.

Figura 3. Total de número de casos de síndrome respiratória aguda grave (SRAG) em indígenas e, dentre estes, quantidade de indivíduos que fizeram uso de suporte ventilatório, foram internados em unidades de terapia intensiva (UTI) e/ou evoluíram a óbito, de 1 de janeiro a 16 de junho de 2017, 2018, 2019 e 2020.

respiratória como causa básica de morte nas declarações de óbito registradas ${ }^{14,15,16}$. Os estudos realizados por Alves et al. ${ }^{15}$ e Bastos et al. ${ }^{16}$ sugeriram a possibilidade de subnotificação de casos da COVID-19 no país, frequentemente notificada de outras formas, como SRAG sem teste laboratorial para COVID-19, o que pode ser observado com o aumento significativo do número de casos e óbitos de pacientes com essa síndrome em 2020 quando comparado aos anos anteriores, mesmo após a exclusão dos casos identificados com o vírus SARS-CoV-2. No Brasil, o aumento do número de óbitos por SRAG é impulsionado pelas precárias condições de trabalho nas urgências e emergências, pelo mau preenchimento da declaração de óbito e pela pouca ou inexistente assistência médica durante a doença terminal ${ }^{14}$. Dessa forma, os casos e mortes declarados como outras causas podem afetar o conhecimento concreto da incidência e da mortalidade pela COVID-19, tanto no cenário brasileiro, quanto no cenário da população indígena.

Na população indígena em 2020 houve 688 casos de SRAG, com $46,22 \%$ desses confirmados para COVID-19. Os indígenas que contraíram o SARS-CoV-2 evoluíram ao óbito em $48,74 \%$ dos casos, o que representa letalidade de 48,74 com incidência de 38,87 contra letalidade de 23,23 e incidência de 138,53 da população total brasileira ${ }^{10}$.

Por décadas, em um passado recente, as populações indígenas foram devastadas por doenças infecciosas, como o sarampo, por não serem previamente sensibilizadas ${ }^{17}$. Atualmente, a COVID-19 é uma novidade para todo o mundo ${ }^{7,17}$, o que não impede, entretanto, que esses indivíduos se mantenham vulneráveis à situação, visto que estão sujeitos a outros fatores, como a iniquidade de fatores médicos, sociais e ambientais,

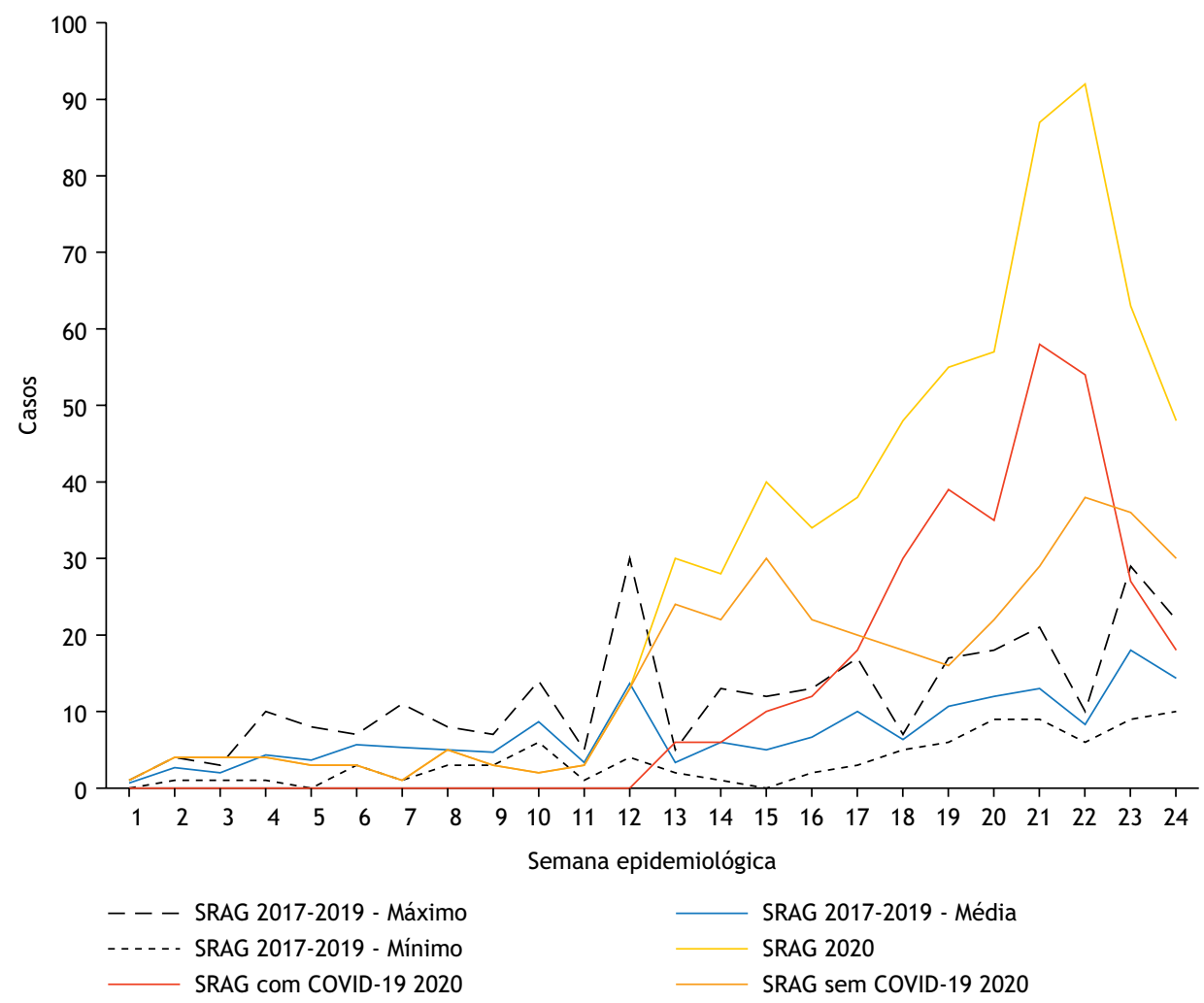

Fonte: Dados do SIVEP-Gripe da SVS/MS do Brasil, 2020.

Figura 4. Total dos casos de síndrome respiratória aguda grave (SRAG) entre indígenas e medidas de média, mínimo e máxima segundo a semana epidemiológica, da $1^{\mathrm{a}}$ a 24ª , para 2017-2019, e incluindo a COVID-19, 2020. 
a alta prevalência de tuberculose e malária, a falta de fonte de água potável, a insuficiência de territórios reconhecidos, a insegurança alimentar, as condições precárias de vida e até a presença de garimpeiros e missionários evangélicos, que podem servir de vetores de transmissão da doença ${ }^{7,17,18}$. Ademais, essas populações têm renda mais baixa e menores níveis de escolaridade, além de sofrerem com resquícios do racismo estrutural e da discriminação histórica ${ }^{19}$.

A região da Amazônia Legal, que conta com os estados do Amazonas, Pará e Roraima e abriga grande quantidade de povos indígenas, apresenta maior proporção de fontes alternativas de água, baixo acesso à eletricidade e ao banheiro ou sanitário, contando frequentemente com o uso de recursos naturais e maior grau de isolamento, o que poderia ser um fator protetor ${ }^{1}$. No entanto, o menor acesso à energia elétrica, o consumo de bens e serviços e o próprio distanciamento de cidades e piores condições de comunicação podem favorecer a identificação e o transporte tardio dos casos dos doentes ${ }^{1,18}$.

É interessante observar que a Amazônia Legal apresenta a maior proporção do país de população indígena rural em municípios com probabilidade alta da epidemia ${ }^{1}$. Vale ressaltar que Amazônia e Pará, até 28 de agosto de 2020, ocupavam espaço entre os dez estados com maior número de casos de COVID-19, estando a Amazônia em $8^{\mathrm{a}}$ posição, com 148.923 casos e o Pará em $6^{\mathrm{a}}$ posição, com 196.874 infectados $^{6}$.

Além disso, na região amazônica, onde existe grande presença indígena, há menor número de cidades com hospitais equipados com UTI, esses geralmente já ocupados, e um número consequentemente limitado de leitos e ventiladores ${ }^{5,17,18,20}$, além da região ter um quarto da população em localidades rurais, incluindo indígenas, com o menor índice de desenvolvimento humano do país ${ }^{5}$. As regiões Norte e Nordeste do país, nos informes epidemiológicos disponibilizados pela Secretaria Especial de Saúde Indígena (SESAI), apresentaram a maior taxa de crescimento e menor tempo de duplicação (tempo necessário para que a doença duplique seu número de casos) da COVID-19, ratificando as iniquidades socioeconômicas da região ${ }^{21}$.

Houve quantidade considerável de casos e óbitos entre indígenas por SRAG também no estado de São Paulo, pertencente à região Sudeste, onde há elevado acesso à eletricidade, à água de poço ou rede geral e maior proporção de utilização de banheiro ou fossa séptica. Esses fatores podem contribuir com a maior proximidade dos indígenas às áreas urbanas e, embora contribuam com melhores condições de vida, também aumentam o risco de transmissibilidade da doença' ${ }^{1}$. São Paulo, até 28 de agosto de 2020, era o estado com maior número de casos e abrigava, inclusive, a terra indígena (TI) Jaraguá, que tem maior índice de vulnerabilidade do país, pois, além da marginalização social e confinamento territorial, tem $18 \%$ de sua população acima dos 60 anos $^{6,18}$. É importante ressaltar que o desaparecimento da população idosa nas comunidades indígenas, seja pela COVID-19 ou por quaisquer outras condições assimiladas pela calamitosa vulnerabilidade social, geográfica e econômica, representa irreversíveis consequências à integridade cultural indígena ${ }^{18}$.
0 fluxo de indígenas para centros regionais que concentram serviços e comércio, onde a frequência de casos pode ser maior, amplia a vulnerabilidade dessa população', uma vez que, geralmente, o acesso a serviços básicos acontece somente nas áreas urbanas, e, inclusive, com deficiente infraestrutura para atender toda a demanda ${ }^{5}$. Moradores rurais entram em contato com pelo menos uma pessoa infectada $(\mathrm{PCl})$ ao visitar uma cidade, uma variável criada que demonstra a importância do fluxo de índios para cidades durante a pandemia. A PCI foi criada para entender o processo de disseminação da doença nos municípios do interior do Amazonas mediante aplicação de cenários distintos de distanciamento social e fluxo de pessoas e verificou-se que as medidas de distanciamento social, o número de visitas às cidades e a duração das mesmas são preponderantes na infecção dos visitantes ${ }^{5}$.

O pacote emergencial de ajuda financeira adotado recentemente pelo governo brasileiro, de 600 reais por pessoa por mês, que abarca várias comunidades indígenas, só pode ser coletado nas cidades, o que força populações indígenas a deixar suas aldeias e serem potencialmente infectadas nos centros urbanos ${ }^{17}$. A Fundação Nacional do Índio (Funai) lançou, inclusive, uma cartilha orientando a população indígena a não sair de suas aldeias sem necessidade ${ }^{22}$. 0 estudo de Ramalho et al. ${ }^{5}$ mostrou que os pontos de distribuição do auxílio, bem como de provisões, mercados e bancos, são locais de aglomeração de pessoas, mesmo mediante medidas de isolamento social impostas localmente. Ademais, um modelo matemático mostrou que residentes ou não de TI próximas a centros urbanos, como Manaus, Fortaleza, Salvador, Boa Vista, Belém e capitais do Sul e Sudeste, incluindo São Paulo e Belo Horizonte, apresentaram maior probabilidade de disseminação da doença ${ }^{1}$, uma vez que essas cidades estão no ranking das 20 cidades brasileiras com maior número de $\operatorname{casos}^{6}$, o que também condiz com a distribuição geográfica obtida neste estudo de casos e óbitos por SRAG entre indígenas na região Sul.

Quanto à zona de residência dos indivíduos acometidos, a SRAG apresentou $2,33 \%$ e $1,42 \%$ a mais de casos e óbitos, respectivamente, na zona rural, o que não representou diferença significante estatisticamente, enquanto na COVID-19 predominaram, por $13,83 \%$ e $5,16 \%$, os casos e óbitos urbanos (Tabela 1). A quantidade de casos e óbitos urbanos pela COVID-19 pode ser explicada pela maior facilidade dessas regiões, especialmente grandes centros urbanos, de transmissibilidade viral, uma vez que o SARS-CoV-2 é transmitido de pessoa para pessoa através de gotículas expelidas, bem como por meio de fômites ${ }^{23}$, esses muito mais presentes no meio urbano. É válido lembrar que as cidades também apresentam maior densidade demográfica, maior fluxo comercial, de serviços e de pessoas ${ }^{1}$, assim como maior facilidade de coleta de material biológico para testagem, reforçando a predominância de casos e óbitos urbanos pela COVID-19, enquanto os residentes rurais ficam mais sujeitos ao diagnóstico genérico de SRAG.

Os resultados demonstraram que, em relação à faixa etária, houve um pico de casos e óbitos por SRAG entre os menores de 1 ano e outro entre os maiores de 50 anos. Para a COVID-19, os picos aconteceram em menores de 1 ano e maiores de 60 anos (Tabela 1). 
A infecção pelo SARS-CoV-2 apresenta maior proporção de casos graves em maiores de 55 ou 60 anos ${ }^{23,24,25}$, o que vai ao encontro dos resultados encontrados. Além disso, casos mais severos também estão relacionados com comorbidades crônicas cardiovasculares e cerebrovasculares ${ }^{23,24}$. Controversamente, um número reduzido de casos tem sido encontrado em menores de 15 anos e quando encontrados apresentam sintomas geralmente mais brandos e com um bom prognóstico, mesmo na presença de opacidade pulmonar nos exames radiológicos ${ }^{23,24,26}$. A literatura mostra que a COVID-19 apresenta mortalidade e gravidade semelhante à SRAG em pacientes pediátricos e que crianças de todas as idades podem ser infectadas pelo SARS-CoV-2, porém são menos afetadas $^{26}$. Existe a possibilidade, evidentemente, de subnotificação de casos nessa faixa etária justamente pelos sintomas serem mais brandos e os responsáveis não procurarem auxílio médico, somando-se aos fatos já elencados de menor comunicação e identificação de casos entre indígenas ${ }^{23}$.

Em pacientes pediátricos, a principal forma de infecção é por contato com familiares infectados ${ }^{26}$, fator preponderante nas comunidades indígenas pelas residências contarem com alto número de moradores ${ }^{7}$. Dentro da faixa etária pediátrica, estudos mostraram que a taxa de hospitalização em cuidados intensivos foi em torno de $4 \%$, sendo $80 \%$ desses de menores de 1 ano de idade ${ }^{26}$, o que corrobora com os resultados encontrados. Entretanto, isso não ratifica o pico de letalidade, o que levanta a hipótese de que a alta dos casos na faixa etária menor de 1 ano é devida, além da subnotificação vigente, às vulnerabilidades socioeconômicas e geográficas supracitadas. Adicionalmente, a correlação entre idade e letalidade encontrada na população indígena segue a tendência da literatura, com aumento crescente no risco de mortalidade no decorrer das faixas etárias, com o maior risco encontrado naqueles com mais de 85 anos $^{23,24}$.

A diferença observada tanto na SRAG, com 57,99\% de casos masculinos, quanto na COVID-19, com $61,32 \%$, para a diferença entre os sexos vai ao encontro da literatura científica, onde os homens têm a tendência de serem mais infectados, de apresentarem formas mais severas da COVID-19 e, consequentemente, de evoluírem mais ao óbito, caracterizando o sexo masculino no contexto da infecção por SARS-CoV-2 como fator de risco tanto para incidência, quanto para mortalidade ${ }^{25,27}$.

Existem contradições na literatura em definir a exata fisiopatogênese e o papel dos principais autores envolvidos na diferença epidemiológica entre os sexos. No entanto, os receptores da enzima conversora de angiotensina 2 (ECA2), que participam do sistema renina-angiotensina-aldosterona, têm papel importante, uma vez que é a ferramenta utilizada pelo vírus para entrar nas células hospedeiras, e sofre, inclusive, influência dos hormônios sexuais ${ }^{24,25,27,28,29}$. Além disso, mulheres apresentam melhor reconhecimento e modulação da resposta imunológica do que homens ${ }^{25}$. No contexto da COVID-19, as diferenças de gênero, normas, papéis, atribuições e comportamentos culturais e sociais também estão envolvidos, homens geralmente apresentam maiores taxas de comorbidades, mais comportamento de risco, como o tabagismo e etilismo, e são menos aderentes às medidas para evitar a transmissão viral, tais como lavar as mãos, rejeitar o isolamento social e outras variáveis socioeconômicas ${ }^{25}$.

O interessante é que a hipótese relacionada aos receptores de ECA2 também é válida e ajuda a explicar a maior mortalidade relacionada com o aumento da idade: idosos teriam expressão diferente de ECA2 e, por isso, maior letalidade associada ${ }^{29}$. Não podemos esquecer, evidentemente, que idosos são epidemiologicamente maiores portadores de doenças crônicas ${ }^{30}$, o que também ajuda a explicar a maior letalidade nessa faixa etária, principalmente quando existem patologias cardiovasculares envolvidas, associadas a pior prognóstico ${ }^{25}$.

O Plano de Contingência Nacional para Infecção Humana pelo SARS-CoV-2 em Povos Indígenas leva em consideração as especificidades da atenção à saúde indígena e apresenta medidas como fortalecimento da detecção, notificação e monitoramento de casos suspeitos, ações de prevenção e controle de infecção, assistência farmacêutica para distribuição e estoque estratégico de medicamentos e suporte laboratorial com a garantia dos fluxos de diagnóstico para os diferentes níveis de resposta: alerta, perigo iminente e emergência em saúde pública ${ }^{31}$.

É possível relacionar o plano de contingência nacional com os informes epidemiológicos disponibilizados pela SESAI por meio das variáveis: número de reprodução efetivo (Re), taxa de crescimento e tempo de duplicação ${ }^{21}$. O número de Re pode ser explicado como o número de casos secundários gerados por um caso primário, sendo que valores $>1$ indicam que há transmissão ativa $^{32}$. A taxa de crescimento representa o incremento de casos por dia e o tempo de duplicação é o número de dias que a série atual de casos duplica nas comunidades indígenas ${ }^{32}$.

Em todo o período analisado, os informes epidemiológicos apresentaram diminuição no Re, sendo de 1,43 no informe da SE 24, primeiro informe com essa variável, e de 1,33 no informe da SE 30, com mínima de 1,29 no informe da $26^{\mathrm{a}} \mathrm{SE}^{21}$. Dessa forma, todos os valores indicam transmissão ativa de COVID-19 nos Distritos Sanitários Especiais Indígenas (DSEI). Ademais, a taxa de crescimento passou de 5,3 casos/dia para 6,3 e o tempo de duplicação de 13 para 11 dias da SE 28 para a 3021, o que indica que a COVID-19 cresce em ritmo mais acelerado e duplica a quantidade de casos em menor tempo. Esses dados ratificam o crescimento da doença mesmo concomitante à aplicação das medidas do plano de contingência nacional, o que levanta a hipótese de baixa eficiência na execução das medidas propostas.

Ressalta-se que, no boletim epidemiológico de 16 de junho de 2020 da SESAl ${ }^{21}$, havia registro de apenas 103 óbitos pela COVID-19 entre indígenas, enquanto nos dados apresentados neste trabalho, com análise até a mesma data, havia registro de 155 óbitos para pacientes com a SRAG e a COVID-19 confirmada, 52 a mais do que o registrado no boletim, número que foi superado pelos boletins apenas em 30 de junho, após 14 dias. No país, a chegada do novo vírus coloca em prova a estrutura de vigilância e sua capacidade de detecção e resposta precoces ${ }^{33}$. Frequentemente desabastecido com a falta de kits para detecção e pessoal capacitado, o Brasil sofre com atraso na liberação 
de resultados de exames, sobrecarga de laboratórios de referência e, por fim, atraso na notificação ${ }^{33}$. Em muitas localidades do país, inclusive, ainda são utilizadas fichas de notificação em papel, que geram não só demora na passagem de dados para os sistemas, como levam a entrada de dados incorretos que poderiam ser automaticamente corrigidos caso tais fichas fossem preenchidas diretamente em seus formulários eletrônicos ${ }^{33}$. A diferença considerável entre o número de óbitos registrados por diferentes informes do governo revela a necessidade de reestruturação da forma como doenças são notificadas no país, visto que, segundo o Guia de Vigilância em Saúde (2019) do MS, constitui parte da Vigilância em Saúde efetiva durante uma pandemia a divulgação de dados com a maior brevidade possível ${ }^{9}$, principalmente devido ao fato de que pandemias tem ocorrido com maior frequência ${ }^{33}$. De acordo com a Organização Mundial da Saúde (OMS), é importante a preparação antecipada das diferentes nações para a emergência de novos patógenos e, para tal, seria essencial a adoção de um sistema que possa notificar e veicular as informações de forma mais célere e confiável ${ }^{33}$.

Ressalta-se, por fim, que o papel da Vigilância em Saúde durante surtos e epidemias é o de identificar fontes de infecção e modos de transmissão, confirmar quantidade de casos e óbitos e diagnósticos laboratoriais, bem como determinar condições relacionadas à propagação de doenças em grupos expostos a maiores riscos ou fatores de risco ${ }^{9}$, reforçando a necessidade da produção de dados atualizados e fidedignos, de forma a proteger a população indígena vulnerável e preservar os modos de vida de seus povos, visto que o principal objetivo da investigação de uma epidemia é o de identificar formas de interromper e prevenir a ocorrência de novos casos 9 . Nesse sentido, cabe elencar uma limitação relevante da análise: o levantamento populacional dos povos indígenas mais recente é o divulgado pelo censo demográfico realizado em 2010, pelo IBGE, o que prejudica a análise e o acompanhamento efetivo dessa população pelos órgãos de Vigilância em Saúde, dificultando o dimensionamento fidedigno do impacto da pandemia entre esses povos.

É evidente que medidas para mitigar a transmissão da COVID-19 devem, portanto, ser propostas. Contudo, é vital que a heterogeneidade e diversidade de contextos regionais das populações indígenas, bem como perfis epidemiológicos, sejam considerados ${ }^{18,19}$. O controle da entrada de pessoas, mesmo assintomáticas em $\mathrm{TI}$, a garantia ao acesso às ações de saúde, à alimentação adequada e a implementação de ações de controle e vigilância da doença com lideranças e organizações indígenas são soluções propostas na literatura ${ }^{7}$. Ademais, evitar aglomerações e contato físico, implantar leitos e remanejar pacientes em UTI ${ }^{18}$, reduzir o número e tempo de permanência das visitas aos centros urbanos e, por fim, coordenar informações sobre medidas a serem tomadas pelas famílias a fim de se protegerem do vírus ${ }^{15}$, inclusive com as orientações necessárias caso algum membro da família adoeça, são, da mesma forma, relevantes ${ }^{19}$.

\section{CONCLUSÕES}

O presente estudo analisou o impacto da pandemia da COVID-19 na população indígena brasileira durante o ano de 2020 por meio das internações por SRAG entre esses indivíduos. A análise foi feita por meio de dados secundários, sujeitos a erros e vieses de preenchimento, bem como à subnotificação.

Foi identificado um importante impacto de casos e óbitos produzidos pela SRAG e pela SRAG com diagnóstico etiológico da COVID-19 nesses povos. Homens, maiores de 50 anos, moradores de áreas urbanas e rurais de populações indígenas dos estados do Amazonas, São Paulo, Pará e Roraima foram os mais frequentemente acometidos.

Por fim, percebe-se que as populações indígenas espalhadas pelo Brasil sofrem as consequências de diversos fatores, que as colocam em situações de maior vulnerabilidade durante a pandemia, pondo em risco não só sua saúde, como também o patrimônio cultural e conhecimentos de gerações passadas. Faz-se necessária, dessa forma, a realização de ações que levem em consideração as particularidades desses povos, de modo que a prevenção, o monitoramento e as medidas de controle e tratamento sejam efetivados. Mostra-se, portanto, ser de suma importância que dados atualizados sobre a população brasileira de indígenas sejam levantados com maior frequência e que outros estudos com essa população sejam realizados, buscando-se entender melhor a dinâmica de saúde indígena em meio à pandemia e formas de intervir nesse processo.

\section{REFERÊNCIAS}

1. Fundação Oswaldo Cruz - Fiocruz. Risco de espalhamento da COVID-19 em populações indígenas: considerações preliminares sobre vulnerabilidade geográfica e sociodemográfica. Rio de Janeiro: Fundação Oswaldo Cruz; 2020[acesso 29 ago 2020]. Disponível em: https://agencia.fiocruz.br/sites/agencia. fiocruz.br/files/u91/relatorios_tecnicos_-_covid-19_proccemap-ensp-covid-19-report4_20200419-indigenas.pdf

2. Ministério da Saúde (BR). Boletim epidemiológico especial semana epidemiológica 23. Brasília: Ministério da Saúde; 2020[acesso 29 ago 2020]. Disponível em: https: //saude.gov.br/images/pdf/2020/August/27/Boletimepidemiologico-COVID-28-FINAL-COE.pdf
3. Ministério da Defesa (BR). Situação do COVID-19 na Amazônia legal. Brasília: Ministério da Defesa; 2020[acesso 29 ago 2020]. Disponível em: http://www.sipam.gov.br/ assuntos/mapas-covid-19-amazonia-legal/covid19_amz_ legal_br_mundo_22ago20-1.pdf

4. Instituto Brasileiro de Geografia e Estatística IBGE. Censo demográfico 2010: características gerais dos indígenas, resultados do universo. Rio de Janeiro: Instituto Brasileiro de Geografia e Estatística; 2020[acesso 29 ago 2020]. Disponível em: https: / /biblioteca.ibge.gov.br/visualizacao/ periodicos/95/cd_2010_indigenas_universo.pdf 
5. Ramalho EE, Junqueira I, Baccaro F, Hill AL, Martins MIFPO, Barcelos DC et al. Dissemination of COVID-19 in cities and riverine communities in central Amazonia. Scielo Preprints. 2020:1-18. https://doi.org/10.1590/SciELOPreprints.406

6. Cota W. Monitoring the number of COVID-19 cases and deaths in Brazil at municipal and federative units level. Scielo Preprints. 2020:1-7. https://doi.org/10.1590/SciELOPreprints.362

7. Associação Brasileira de Saúde Coletiva - Abrasco. A COVID-19 e os povos indígenas: desafios e medidas para controle do seu avanço. Rio de Janeiro: Associação Brasileira de Saúde Coletiva; 2020[acesso 28 ago 2020]. Disponível em: https://www.abrasco.org.br/site/noticias/ posicionamentos-oficiais-abrasco/a-covid-19-e-os-povosindigenas-desafios-e-medidas-para-controle-do-seuavanco/45866/

8. Silva WNT, Rosa MFP, Oliveira SV. Produção de boletins epidemiológicos como estratégia de vigilância em saúde no contexto da pandemia de COVID-19. Vigil Sanit Debate. 2020;8(3):171-7. https://doi.org/10.22239/2317-269x.01658

9. Ministério da Saúde (BR). Guia de vigilância em saúde. Brasília: Ministério da Saúde; 2019.

10. Ministério da Saúde (BR). Plataforma integrada de vigilância em saúde: dados abertos. Brasília: Ministério da Saúde; 2020[acesso 28 ago 2020]. Disponível em: http://plataforma. saude.gov.br/coronavirus/dados-abertos/

11. Instituto Brasileiro de Geografia e Estatística - IBGE. Brasil: cidades. Rio de Janeiro: Instituto Brasileiro de Geografia e Estatística; 2020[acesso 10 ago 2020]. Disponível em: https://cidades.ibge.gov.br/brasil/panorama

12. Instituto Brasileiro de Geografia e Estatística - IBGE. Censo 2010. Rio de Janeiro: Instituto Brasileiro de Geografia e Estatística; 2020[acesso 28 ago 2020]. Disponível em: https://censo2010.ibge.gov.br/

13. Ministério da Saúde (BR). Ficha de registro individual: casos de síndrome respiratória aguda grave hospitalizado. Brasília: Ministério da Saúde; 2020[acesso 28 ago 2020]. Disponível em: http://plataforma.saude.gov.br/ coronavirus/dados-abertos/sivep-gripe/ficha-SIVEP-GRIPESRAG-hospital-Sivepgripe.pdf

14. França EB, Ishitani LH, Teixeira RA. Óbitos por COVID-19 no Brasil: quantos e quais estamos identificando? Rev Bras Epidemiol. 2020;23:1-7. https: //doi.org/10.1590/1980-549720200053

15. Alves THE, Souza TA, Silva SA, Ramos NA, Oliveira SV. Underreporting of death by COVID-19 in Brazil's second most populous state. Medrxiv. 2020:1-15. https://doi.org/10.1101/2020.05.20.20108415

16. Bastos LS, Niquini RP, Lana RM. COVID-19 e hospitalizações por SRAG no Brasil: uma comparação até a $12^{\mathrm{a}}$ semana epidemiológica de 2020. Cad Saude Publica. 2020;36(4):1-8. https://doi.org/10.1590/0102-311x00070120

17. Amigo I. Indigenous communities in Brazil fear pandemic's impact. Science. 2020;368(6489):1-2. https://doi.org/10.1126/science.368.6489.352
18. Oliveira U, Soares Filho B, Oviedo A, Moreira T, Carlos S, Ricardo $\mathrm{J}$ et al. Modelagem da vulnerabilidade dos povos indígenas no Brasil ao COVID-19. São Paulo: Instituto Socioambiental; 2020[acesso 28 ago 2020]. Disponível em: https://www.socioambiental.org/sites/blog. socioambiental.org/files/nsa/arquivos/nota_tecnica_ modelo_covid19.pdf

19. Pan American Health Organization - PAHO. Considerações sobre povos indígenas, afrodescendentes e outros grupos étnicos durante a pandemia de COVID-19. Washington: Pan American Health Organization; 2020[acesso 28 ago 2020]. Disponível em: https: / /iris.paho.org/handle/10665.2/52280

20. Mendonça FD, Rocha SS, Pinheiro DLP, Oliveira SV. Região norte do Brasil e a pandemia de COVID-19: análise socioeconômica e epidemiológica. J Health Npeps. 2020;5(1):20-37. https://doi.org/10.30681/252610104535

21. Ministério da Saúde (BR). Informe epidemiológico SESAI. Brasília: Ministério da Saúde; 2020[acesso 28 ago 2020]. Disponível em: http://www.saudeindigena.net.br/ coronavirus/boletimEp.php

22. Fundação Nacional do Índio - Funai. Como sacar o benefício: auxílio emergencial. Brasília: Fundação Nacional do Índio; 2020[acesso 28 ago 2020]. Disponível em: https://drive.google.com/ file/d/1dW_ujl61GLMUpZ3n_kbeJiQazKpQUdSM/view

23. Harapan H, Itoh N, Yufika A, Keam S, Te H, Megawati D et al. Coronavirus disease 2019 (COVID-19): a literature review. J Infect Public Health. 2020;13(5):667-73. https://doi.org/10.1016/j.jiph.2020.03.019

24. Uddin M, Mustafa F, Rizvi TA, Loney T, Al Suwaidi $\mathrm{H}$, Al-Marzouqi AHH et al. SARS-CoV-2/COVID-19: viral genomics, epidemiology, vaccines, and therapeutic interventions. Viruses. 2020;12(5):1-18. https://doi.org/10.3390/v12050526

25. Gebhard C, Regitz-Zagrosek V, Neuhauser HK, Morgan R, Klein SL. Impact of sex and gender on COVID-19 outcomes in Europe. Biol Sex Differ. 2020;11(29):1-13. https: / /doi.org/10.1186/s13293-020-00304-9

26. Tezer H, Demirdag TB. Novel coronavirus disease (COVID-19) in children. Turk J Med Sci. 2020;50(3):592-603. https://doi.org/10.3906/sag-2004-174

27. Galbadage T, Peterson BM, Awada J, Buck AS, Ramirez DA, Wilson J et al. Systematic review and meta-analysis of sexspecific COVID-19 clinical outcomes. Front Med (Lausanne). 2020;7:1-15. https://doi.org/10.3389/fmed.2020.00348

28. Jin JM, Bai P, He W, Wu F, Liu XM, Han DM et al. Gender differences in patients with COVID-19: focus on severity and mortality. Front Public Health. 2020;8:1-6. https://doi.org/10.3389/fpubh.2020.00152

29. Ambrosino I, Barbagelata E, Ortona E, Ruggieri A, Massiah $G$, Giannico OV et al. Gender differences in patients with COVID-19: a narrative review. Monaldi Arch Chest Dis. 2020;90(2):318-24. https://doi.org/10.4081/monaldi.2020.1389

30. Cockerham WC, Hamby BW, Oates GR. The social determinants of chronic disease. Am J Prev Med. 2017;52(1 supl 1):s5-s12. https://doi.org/10.1016/j.amepre.2016.09.010 
31. Ministério da Saúde (BR). Plano de contingência nacional para infecção humana pelo novo coronavírus (COVID-19) em povos indígenas. Brasília: Ministério da Saúde; 2020[acesso 28 ago 2020]. Disponível em: http://docs.bvsalud.org/ biblioref/2020/04/1095139/plano_de_contingencia_da_ saude_indigena_preliminar.pdf

32. Ministério da Saúde (BR). Informe epidemiológico: doença por coronavírus (COVID-19) em populações indígenas- semana epidemiológica 30. Brasília:
Ministério da Saúde; 2020[acesso 28 ago 2020].

Disponível em: http://www. saudeindigena.net.br/ coronavirus/pdf/Informe\%20Epidemiologico\%20SE\%20 30\%20-\%20SESAI\%20COVID\%2019.pdf

33. Lana RM, Coelho FC, Gomes MFC, Cruz OG, Bastos LS, Villela DAM et al. Emergência do novo coronavírus (SARS-CoV-2) e o papel de uma vigilância nacional em saúde oportuna e efetiva. Cad Saude Publica. 2020;36(3):1-5. https://doi.org/10.1590/0102-311×00019620

Contribuição dos Autores

Silva WNT, Rosa MFP, Mendonça KS, Queiroz GA, Oliveira SV - Concepção, planejamento (desenho do estudo), aquisição, análise, interpretação dos dados e redação do trabalho. Todos os autores aprovaram a versão final do trabalho.

Conflito de Interesse

Os autores informam não haver qualquer potencial conflito de interesse com pares e instituições, políticos ou financeiros deste estudo.

Licença CC BY-NC atribuição não comercial. Com essa licença é permitido acessar, baixar (download), copiar, imprimir, compartilhar, reutilizar e distribuir os artigos, desde que para uso não comercial e com a citação da fonte, conferindo os devidos créditos de autoria e menção à Visa em Debate. Nesses casos, nenhuma permissão é necessária por parte dos autores ou dos editores. 